\title{
Intervenção estética anterior por meio de facetas diretas em resina composta: relato de caso
}

\author{
Previous aesthetic intervention using direct facets in composite resin: case report
}

Intervención estética previa mediante facetas directas en resina compuesta: relato de caso

Aurélio de Oliveira Rocha ${ }^{1 *}$, Lucas Menezes dos Anjos², Maria de Nazaré Oliveira Rocha ${ }^{3}$, Thaine Oliveira Lima ${ }^{1}$, Priscilla Castro Moura Rodrigues ${ }^{1}$, Lucas Ferreira de Sá Santos ${ }^{1}$,Rafaela de Menezes dos Anjos Santos ${ }^{2}$, Ingrid de Melo Silva ${ }^{1}$, Denílson Oliveira Correia da Silva ${ }^{1}$, William José e Silva Filho².

\section{RESUMO}

Objetivo: Descrever um caso clínico de intervenção restauradora nas unidades dentais anteriores superiores com facetas em resina composta e discutir a cerca desse tratamento. Detalhamento do caso: Paciente procurou atendimento no consultório odontológico queixando-se da harmonia do seu sorriso, onde o mesmo não gostava dos dentes amarelados, pequenos, separados e levemente desalinhados. Ao exame clínico verificou-se a presença de diastemas interdentais, unidades 12 e $22 \mathrm{com}$ anatomia conóide e unidades 13 e 23 levemente girovertidos para distal. Tendo em vista as limitações do paciente e análise clínica, foi proposto reabilitação estética e funcional das unidades por meio de clareamento dental imediato em consultório, seguido de facetas em resina composta das unidades relatadas. Previamente ao tratamento restaurador foi confeccionado enceramento diagnóstico para evidenciar um possível resultado, planejar com segurança e confeccionar a guia palatina. Considerações finais: Para realização de múltiplas restaurações estéticas é de suma importância conhecimento teórico e prático por parte do cirurgião-dentista, planejamento minucioso do caso por meio do enceramento diagnóstico e cuidados pós-operatórios por parte do paciente, garantido assim o sucesso desse tratamento.

Palavras-chave: Estética dental, Facetas dentárias, Resinas compostas.

\begin{abstract}
Objective: To describe a clinical case of restorative intervention in upper anterior dental units with composite resin veneers and to discuss this treatment. Case details: The patient sought care at the dental office complaining about the harmony of his smile, where he did not like his yellow, small, separated and slightly misaligned teeth. Clinical examination revealed the presence of interdental diastemas, units 12 and 22 with conoid anatomy and units 13 and 23 slightly rotated to the distal. In view of the patient's clinical limitations, aesthetic and functional rehabilitation of the units was proposed by means of immediate dental whitening in the office, followed by facets in composite resin from the reported units. Prior to the restorative treatment, diagnostic waxing was performed to show a possible result, to plan safely and to prepare the palatal guide. Final considerations: To perform multiple aesthetic restorations, theoretical and practical knowledge on the part of the dentist is of paramount importance, meticulous planning of the case through diagnostic waxing and post-operative care by the patient, thus guaranteeing the success of this treatment.
\end{abstract}

Keywords: Dental aesthetics, Dental veneers, Composite resins.

${ }^{1}$ Universidade Tiradentes (UNIT), Aracaju - SE. *E-mail: aureliorocha2015@gmail.com

2 Universidade Federal de Sergipe (UFS), Aracaju - SE.

${ }^{3}$ Faculdade Maurício de Nassau (UNINASSAU), Arapiraca - AL. 


\section{RESUMEN}

Objetivo: Describir un caso clínico de intervención restauradora en unidades dentales anteriores superiores con carillas de resina compuesta y discutir este tratamiento. Detalles del caso: El paciente acudió al consultorio odontológico quejándose de la armonía de su sonrisa, donde no le gustaban sus dientes amarillos, pequeños, separados y levemente desalineados. El examen clínico reveló la presencia de diastemas interdentales, unidades 12 y 22 con anatomía conoide y unidades 13 y 23 ligeramente rotadas hacia distal. Ante las limitaciones clínicas del paciente, se propuso la rehabilitación estética y funcional de las unidades mediante blanqueamiento dental inmediato en consultorio, seguido de facetas en resina compuesta de las unidades reportadas. Previo al tratamiento restaurador, se realizó un encerado diagnóstico para mostrar un posible resultado, planificar con seguridad y preparar la guía palatina. Consideraciones finales: Para realizar múltiples restauraciones estéticas es de suma importancia el conocimiento teórico y práctico por parte del odontólogo, planificación minuciosa del caso mediante encerado diagnóstico y cuidados postoperatorios por parte del paciente, garantizando así el éxito de este tratamiento.

Palabras clave: Estética dental, Carillas dentales, Resinas compuestas.

\section{INTRODUÇÃO}

A estética é associada ao semblante da beleza em sua forma, contorno, textura e cor, sendo de caráter específico de cada indivíduo. A descrição de um sorriso perfeito está relacionada ao bem-estar físico, psicológico, socioeconômico, assim como, ao sucesso pessoal e profissional; a falta da harmonia no sorriso gera prejuízos emocionais, através de ansiedade causando insegurança, timidez ou mesmo isolamento social (BISPO LB, 2009).

A busca por tratamentos e intervenções estéticas nos consultórios odontológicos é crescente devido principalmente a atender padrões subliminarmente impostos pela sociedade, difundidos pela internet, TV ou revistas. Grande parte dos pacientes que procuram por tratamentos visam não apenas saúde ou restabelecimento de função, mas também reabilitações que influenciem em sua autoimagem, seja por vaidade ou aceitação social (PEREIRA DA, et al., 2016).

Dentes com alteração de forma, cor ou posição na maioria dos casos são julgados como não harmônicos ao sorriso, pois fogem do padrão estético difundido nas mídias sociais (BISPO LB, 2009). Essas situações culminam na redução da qualidade de vida dos indivíduos que buscam tratamentos reabilitadores para melhorar as características do sorriso (RODRIGUE SDR, et al., 2014).

Para contornar essa situação o cirurgião-dentista pode lançar mão de diversos tratamentos através de reconstruções indiretas em cerâmica, semi-direta em resinas e diretas em resina composta, essa última acaba sendo a escolha na maioria dos casos, pois apresentam como vantagens a reabilitação imediata, facilidade de reparo e menor custo (HIRATA R, 2016).

Apesar do material restaurador cerâmico ser muito indicado para os tratamentos restauradores estéticos, por serem mais resistentes à fratura e proporcionarem estabilidade da cor, as facetas de resina composta possibilitam reparação da restauração e preparação menos agressiva ao dente (GUERRA M, et al., 2017). Além disso, as facetas diretas podem ser realizadas em sessão única, pois não requerem moldagens e confecção de provisório, dispensando as etapas laboratoriais, consequentemente proporcionando ao paciente um menor custo de tratamento (MACHADO AC, et al.,2016).

Para garantir um melhor resultado, os materiais restauradores vêm obtendo novas características para mimetizar a estrutura dental com maiores detalhes, aproximando o máximo do natural (GOYATÁ FDR, et al., 2017). As resinas compostas da atualidade classificadas de acordo com o tamanho das partículas como nanoparticuladas ou nanohíbridas, proporcionam excelentes características de opalescência e fluorescência dos dentes restaurados. Além da gama de cores que variam de translucidas para opacas (SILVA GR, et al., 2015). 
De acordo com as limitações dos materiais restauradores, é de suma importância o conhecimento atual por parte do cirurgião-dentista em relação aos materiais e técnicas utilizadas, para assim garantir o sucesso funcional e estético do tratamento (GOYATÁ FDR, et al., 2017; RODRIGUE SDR, et al., 2014).

Dentre as etapas prévias a essa intervenção destaca-se o clareamento dental que é indicado quando há uma alteração de cor expressiva que pode interferir no resultado do tratamento, bem como o enceramento diagnóstico que é um passo imprescindível no pré e trans operatório (MARAN BM, et al., 2019; WITTMANN $\mathrm{K}$, et al., 2013). O clareamento dental é um procedimento conservador e que não impossibilita outras formas de tratamento, assim é em muitos casos indicado previamente ao procedimento restaurador, favorecendo para uma tonalidade mais clara do substrato o que favorece na seleção da cor e reduz a necessidade de desgaste (QUAGLIATTO PS, 2020).

O enceramento diagnóstico é um método atualmente empregado entre os cirurgiões-dentistas e considerado imprescindível para a finalidade de orientar a reabilitação oral do paciente, e que irá auxiliar o profissional no planejamento e confecção das facetas dentais diretas (GUERRA M, et al.,2017).

Um planejamento reabilitador estético e funcional envolvem a integração de diversas especialidades da odontologia (MACIELCM, et al., 2020). A evolução do conhecimento e diagnóstico, bem como o desenvolvimento das técnicas e materiais restauradores, gera novas perspectivas que devem ser sempre atualizadas e confrontadas (MACIELCM, et al., 2020). Dentro desta expectativa, é fundamental um planejamento adequado e o conhecimento de novas técnicas por parte dos cirurgiões-dentistas (VIANA PC, et al., 2012).

Assim, o objetivo desse estudo é relatar um caso clínico onde alterações de coloração, posição e formato dental foram corrigidos por meio de clareamento dental, enceramento diagnóstico e aplicação de facetas diretas em resina composta.

\section{DETALHAMENTO DO CASO}

Paciente do sexo masculino, 28 anos, leucoderma, procurou atendimento no consultório odontológico queixando-se da harmonia do sorriso devido coloração amarelada, presença de espaços interdentais, dentes pequenos e mal posicionados. Ao exame clínico foi observado que o mesmo apresentava múltiplos diastemas entre as unidades anteriores superiores, caninos superiores apresentavam leve giroversão para distal e os incisivos laterais apresentavam anatomia conóide (Figura 1).

Figura 1 - Exame clínico inicial do sorriso.

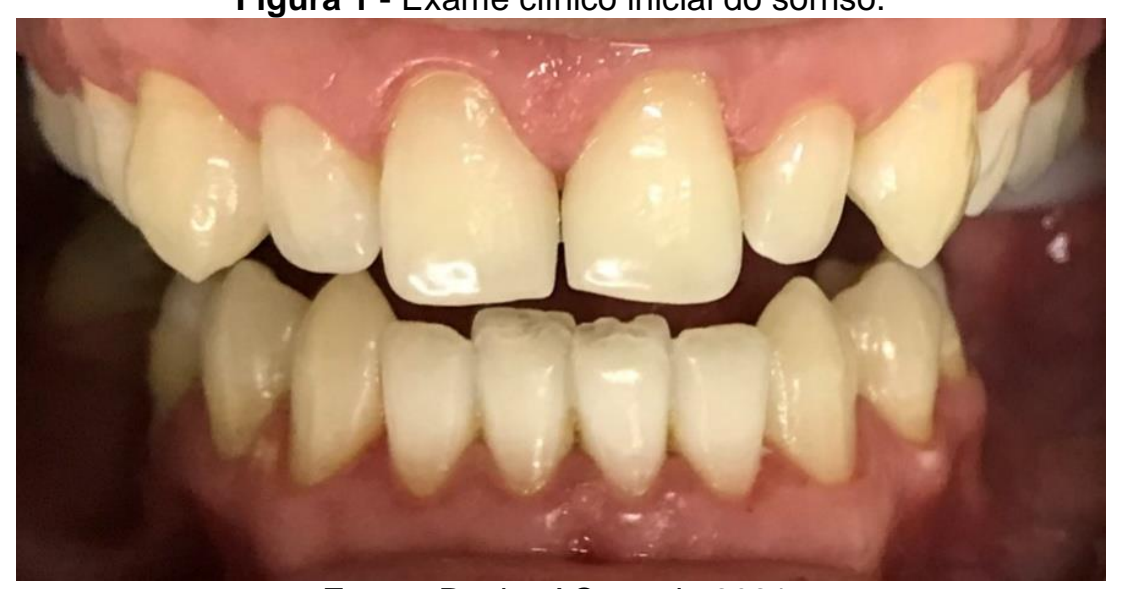

Fonte: Rocha AO, et al., 2021.

Levando em consideração a queixa do paciente, análise oral clínica e limitações financeiras, foram propostas as seguintes etapas de tratamento para o paciente: clareamento dental imediato realizado em consultório que inicialmente compreendia três sessões, confecção de enceramento diagnóstico para posterior realização de facetas diretas em resina composta. 
Foi realizada profilaxia das unidades com pedra pomes (SS WHITE®) e água, seleção de cor de acordo com a escala vita classical $(\mathrm{VITA} \AA)$ onde foram registradas cores DA3 e EA3, aplicação do dessensibilizante Desensibilize $\mathrm{Kf}$ 0,2\% (FGM®)por dez minutos para previnir o risco de sensibilidade pós-operatória, e em seguida foi realizado isolamento relativo com abridor bucal e aplicação da barreira gengival TOP DAM (FGM®), assim foi aplicado o gel clareador Whiteness HP 35\%(FGM®) na superfície, onde este foi mantido por 40 minutos (Figura 2). O clareamento dental foi então realizado em três sessões, pois devido o substrato alcançar o ponto de saturação não houve melhora significante da segunda para a terceira fase.

Figura 2 - Trans-operatório do clareamento dental.

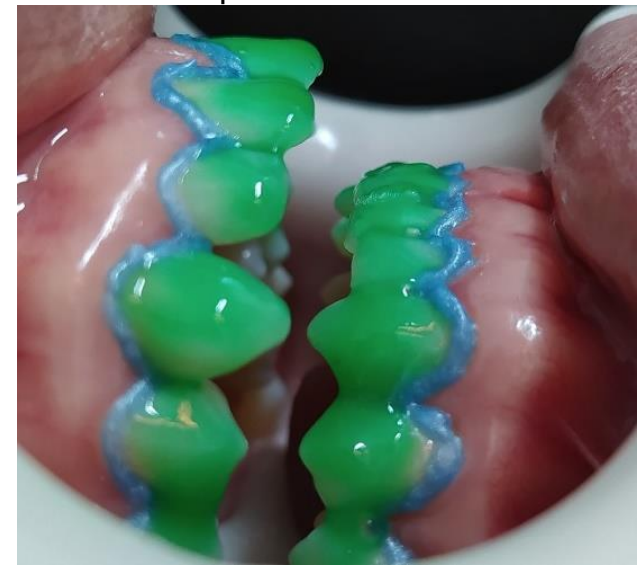

Fonte: Rocha AO, et al., 2021.

Após a terceira sessão do clareamento foi evidenciada cor DA1 e EA1 de acordo com a escala vita classical (VITA®) mostrando uma melhora relevante na coloração, foi então realizada moldagem da arcada superior com alginato (DENTSPLY®) e vazada com gesso especial (DENTSPLY®), dando origem assim ao modelo de trabalho onde foram realizados desgastes estratégicos e confeccionado o enceramento diagnóstico com o auxílio de gotejadores e esculpidores dentais (Figura 3). Esta ferramenta teve como finalidade mostrar ao paciente como seu sorriso possivelmente ficaria em relação a forma e anatomia, além de ser utilizado para confecção da guia palatina com silicona de condensação perfil (COLTENE®).

Figura 3 - Enceramento diagnóstico confeccionado.

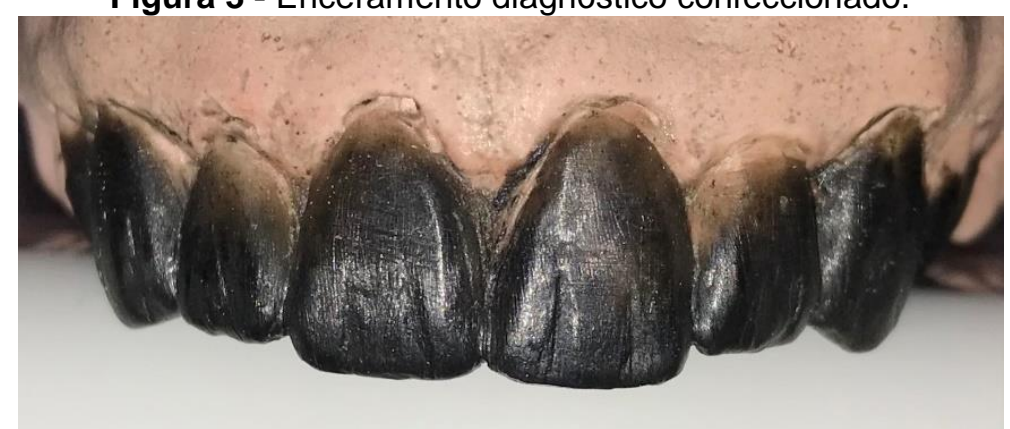

Fonte: Rocha AO, et al., 2021.

A sessão do tratamento restaurador, realizada 15 dias após o clareamento, iniciou-se através de profilaxia com escova de robbinson (PREVEN®) e pasta profilática sem óleo (MAQUIRA®), seleção de cor com escala vita classical $(\mathrm{VITA} \Theta)$ onde as cores escolhidas foram dentina $A 1$, esmalte $A 1$ e translúcida para palatina e incisal. Em seguida com o auxílio de uma ponta diamantada 2200F(KG SORENSEN®) foram realizados pequenos desgastes na mesial dos incisivos laterais para assim criar espaço para aumento da largura dos incisivos centrais e na vestibular dos caninos para posicioná-los corretamente no arco.

Em seguida foi realizado isolamento absoluto modificado e condicionamento com ácido fosfórico $37 \%$ (FGM®) das unidades dentais a serem restauradas por 30 segundos, estas foram em seguida lavadas abundantemente e foi aplicado o adesivo ambar APS (FGM®) que foi fotopolimerizado (SDI®) para assim 
posicionar a guia palatina. Antes do posicionamento da guia foi colocada resina translúcida Harmonize GRY (KERR®) na região além do substrato para criação da muralha palatina que era confeccionada após posicionamento e fotopolimerização $(\mathrm{SDI})$ do material, em seguida foi inserida resina para dentina Vittra APS (FGM®) cor A1 e com o auxílio de esculpidores foi realizada escultura e estratificação da borda incisal (Figura 4), após polimerizar era inserida a resina de esmalte cor A1 Vittra APS (FGM®) em toda a face vestibular da unidade dental, acomodação da resina com pincel pêlo de Marta N.4ª (KOTA®) e escultura com espátulas para assim realizar fotoativação (SDIß) final.

Figura 4 - Escultura incisal para confecção de detalhes anatômicos.

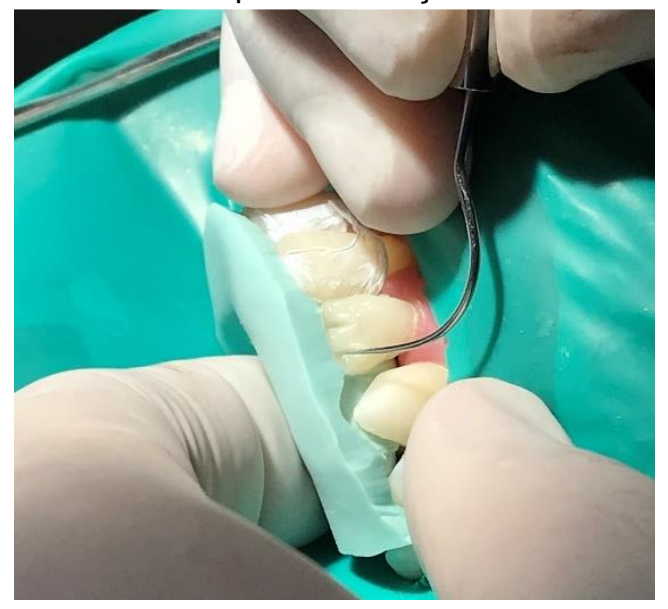

Fonte: Rocha AO, et al., 2021.

Após a remoção do isolamento absoluto foi realizado ajuste oclusal para distribuição de contatos dentais e acabamento das restaurações com brocas multilaminadas(KG SORENSEN®), pontas abrasivas e discos de lixa (AMERICA BURSß) evidenciando o aspecto final imediato das restaurações (Figura 5).No retorno após 7 dias foram realizados ajustes e polimento com disco de feltro (AMERICA BURSß) e pasta para polimento diamond excel (FGM®) (Figura 6).

Figura 5 - Aspecto final imediato das restaurações.

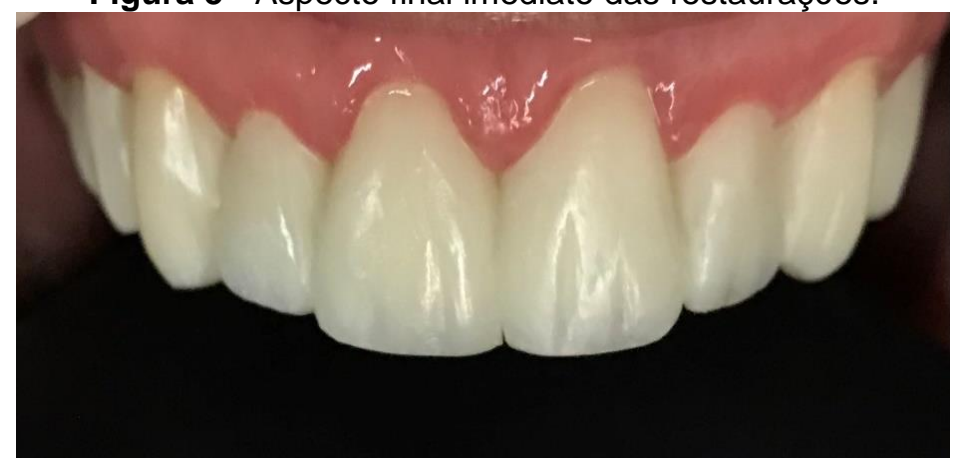

Fonte: Rocha AO, et al., 2021.

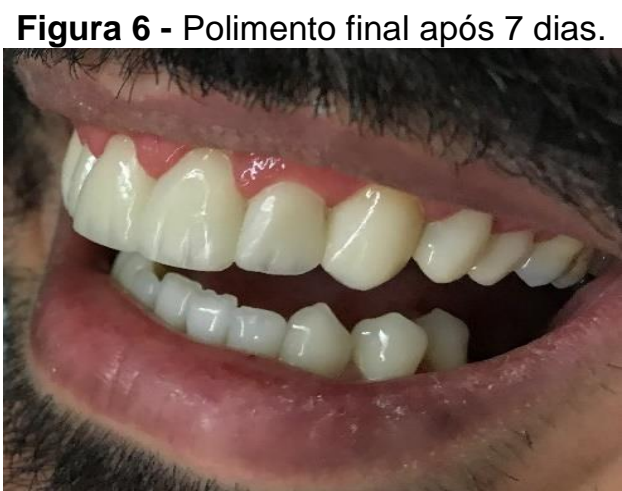

Fonte: Rocha AO, et al., 2021.

\section{DISCUSSÃO}

Reabilitações com finalidade estéticas em odontologia têm se tornado comum por causa do aumento do interesse dos pacientes por tratamentos que afetam a imagem, vaidade e inserção social (WANG C, et al., 2014). Segundo Silva GR, et al. (2015) durante os últimos anos, houve uma revolução tecnológica na interface estética com relação às resinas compostas que permitiu um crescimento e melhoramento de suas propriedades mecânicas, físicas e adesivas (SILVA GR, et al., 2015). Assim, devido a relevância observada nesse material em estudos renomados, este foi o material de escolha para realização da reabilitação estética e funcional nesse caso clínico. 
Os diastemas são caracterizados por espaços interdentais que podem ser causados por diversos fatores como discrepâncias no tamanho dental, ausência de dentes, giroversões, angulações inadequadas entre dentes contíguos, presença de freio lingual com inserção baixa, presença de dentes supranumerários ou problemas periodontais (GUERRA M, et al.,2017). Sendo estes uma das principais queixas do paciente que estavam relacionadas as unidades 12 e 22 com discrepâncias no tamanho do dente apresentando formato conóide e giroversões das unidades 13 e 23.

Segundo Coelho SFH, et al. (2015), as facetas diretas em resina composta são a primeira escolha nos procedimentos que necessitam um mínimo desgaste da estrutura dental, dessa forma, diferindo dos procedimentos indiretos que, além de necessitarem de um desgaste maior da estrutura dentária, tem um custo mais elevado, corroborando com os estudos de Guerra M, et al. (2017) que tiveram as mesmas conclusões. Esse procedimento vem se tornando uma excelente alternativa de tratamento para problemas estéticos em dentes anteriores (KORKUT B, 2018). A aplicação em uma única sessão do material restaurador definitivo com o mínimo de desgaste ou algumas vezes sem nenhum desgaste da superfície dentária é uma das vantagens dessa técnica (KORKUT B, 2018; GUERRA M, et al.,2017).

Entretanto a qualidade em longo prazo desse material tem a limitação de ser totalmente dependente do paciente, pois, se este não tiver uma boa higiene oral, favorecerá a degradação da matriz orgânica da resina e a consequente alteração da sua cor e textura (SILVA GR, et al., 2015). Para contornar essa limitação o paciente foi orientado quanto aos hábitos de higiene e alimentares, bem como ao retorno semestral para realização de manutenção desse material garantindo o sucesso duradouro desse tratamento.

Nos casos que envolvem pacientes jovens, a obtenção do contorno, formato dental, textura das faces lisas e mimetização do substrato dentário é um grande desafio clínico e prático, uma vez que os dentes desse grupo específico possuem grande quantidade de características e detalhes anatômicos (WITTMANN K, et al., 2013). Nestes casos, o esmalte dental possui detalhes de alta opalescência e, a dentina apresenta em sua forma lóbulos evidentes (SENE F, et al., 2016). A borda incisal apresenta alta translucidez com um fino halo opaco (SENE F, et al., 2016). Durante a estratificação das unidades dentais foram utilizadas resinas de efeitos e emprego de técnicas para escultura garantindo assim características mais próximas ao natural.

O tratamento clareador deve ser considerado sempre que tratamentos estéticos anteriores forem propostos ao paciente (MATIS BA, et al., 2015). A existência de restaurações prévias e a possibilidade de novos tratamentos restauradores influenciam no planejamento clareador, visto que restaurações antigas podem tornar-se mais evidentes após o clareamento, enquanto restaurações futuras devem ser confeccionadas baseando-se na cor obtida ao fim desta etapa (HIRATA R, 2016).De acordo com esses estudos, essa etapa foi de grande importância para reduzir drasticamente a necessidade de desgastes e garantir a colocação de uma delgada camada de resina culminando assim em um aspecto natural das unidades dentais e suprindo as expectativas do paciente.

Não foi utilizada fonte de luz, pois as evidências científicas atuais mostram que seu emprego não melhora o efeito clareador (MARAN BM, et al., 2019). Não foi recomendado à paciente evitar o consumo de alimentos e bebidas com corantes, pois os trabalhos clínicos mais recentes não encontraram relação entre seu consumo e a qualidade final do tratamento clareador (REZENDE M, et al., 2013; MATIS BA, et al., 2015; MORI AA, et al., 2016). O intervalo de 2 semanas entre o fim do tratamento clareador e os procedimentos restauradores é importante para garantirmos que o oxigênio residual do clareamento terá sido completamente eliminado (MORI AA, et al., 2016). A realização de procedimentos adesivos antes deste período é um risco, pois tal oxigênio residual pode interferir na adequada polimerização do sistema adesivo (MCGUCKIN RS, et al., 1992; TITLEY KC, et al., 1993). Após o clareamento dental foram esperados 15 dias para assim serem realizados os procedimentos restauradores como recomendado pelos autores citados.

De acordo com Goyatá FDR, et al. (2017) a sessão de acabamento e polimento realizados de forma eficaz e certa, proporciona uma superfície lisa e brilhosa que melhora o contorno da restauração realizada, fornece uma anatomia fiel, aumentando a qualidade e a longevidade das restaurações, indo ao encontro com os estudos de Pereira DA, et al. (2016); Can Say E, et al. (2014). Esse passo foi realizado com o máximo de cuidado utilizando materiais de qualidade e seguindo a ordem de uso de acordo com as recomendações do fabricante, corroborando com os achados literários citados anteriormente. 
A Odontologia vinculada a estética não é atribuição restrita de especialistas, também está presente na prática diária e vivência do clínico geral. Porém, para um resultado final eficiente, além de ser necessária prática e estudo técnico, alguns elementos artísticos voltados à escultura e cor devem ser considerados para indicação e realização do procedimento ideal, pois todo dentista deve realizar um tratamento restaurador o mais próximo possível da dentição humana natural (MACIEL CM, et al., 2020). Os procedimentos reabilitadores estéticos requerem observação, tranquilidade e aplicação cuidadosa das técnicas e protocolos existentes (GUERRA M, et al.,2017). Desta maneira é de responsabilidade do profissional buscar se aperfeiçoar e adquirir conhecimentos, garantindo um resultado natural e harmônico para o sorriso dos pacientes.

\section{REFERÊNCIAS}

1. BISPO LB. Facetas estéticas: Status da arte. Revista Dentística on line, 2009; 8(18): 11-4.

2. CAN SAY E, et al. Surface roughness and morphology of resin composites polished with two-step polishing systems. Dent Mater J. 2014; 33(3): 332-42.

3. COELHO-DE-SOUZA FH, et al. Direct anterior composite veneers in vital and non-vital teeth: A retrospective clinical evaluation. Journal of Dentistry, 2015; 43(11): 1330-1336.

4. GUERRA MLRS, et al. Fechamento de diastemas anteriores com resina composta direta: relato de caso. Faculdade de Odontologia de Lins/Unimep, 2017; 27(1): 63-68.

5. GOYATÁ FDR, et al. Remodelação estética do sorriso com resina composta e clareamento dental em paciente jovem: relato de caso. Arch Heal Investig. 2017;6(9):408-13.

6. HIRATA R. Shortcuts em odontologia estética: uma nova visão sobre TIPS. 1a ed. São Paulo: Quintessence, 2016.

7. KORKUT B. Smile makeover with direct composite veneers: A two-year follow-up report. J Dent Res DentClinDentProspect, 2018; 12(2): 146-151.

8. MACHADO AC, et al. Reabilitação estética e funcional com facetas diretas após histórico de traumatismo dentoalveolar. Rev Odonto Bras Central, 2016.

9. MACIEL CM, et al. Reanatomização e facetas diretas em dentes anteriores: relato de caso. Paraná: Ed Atena, 2020; $416 \mathrm{p}$.

10. MARAN BM, et al. Different light-activation systems associated with dental bleaching: a systematic review and a network meta-analysis. Clinical Oral Investigations, 2019; 23(4): 1499-1512.

11. MATIS BA, et al. White diet: is it necessary during tooth whitening? Operative Dentistry, 2015; 40(3): 235-240.

12. MCGUCKIN RS, et al. Enamel shear bond strengths after vital bleaching. American Journal of Dentistry, 1992; 5(4): 216- 222.

13. MEIRELLES L, et al. Aplicações clínicas do enceramento diagnóstico na reabilitação oral-uma revisão de literatura. Revista da Faculdade de Odontologia de Lins, 2013; 23(1): 20-25.

14. MORI AA, et al. Susceptibility to Coffee Staining during Enamel Remineralization Following the In-Office Bleaching Technique: An In Situ Assessment. Journal of Esthetic and Restorative Dentistry, 2016; 28(1): 23- 31.

15. PEREIRA DA, et al. Reabilitação estética do sorriso por meio de procedimento restaurador direto com resina composta nanoparticulada: relato de caso. Revista Odontológica do Brasil Central, 2016; 25(72): 54-58.

16. QUAGLIATTO PS. Clareamento dental e técnicas restauradoras para dentes clareados. São Paulo: Ed. Santos, 2020. $310 \mathrm{p}$.

17. REZENDE M, et al. Clinical effects of exposure to coffee during at-home vital bleaching. Operative Dentistry, 2013; 38(6): 229-236.

18. RODRIGUE SDR, et al. Reanatomização dental com resina composta. Rev Bahiana Odontol. 2014;5(3):182-92.

19. SENE F, et al. Esthetic remodeling of maxillary incisors with composite resin: color, shape, and proportion correction. J Clin Dent Res. 2016; 13(2): 70-7.

20. SILVA GR, et al. Tratamento estético com diretas de resina composta - relato de caso. Rev UNINGÁ Rev. 2015; 24(3): 27-31.

21. TITLEY KC, et al. Adhesion of a resin composite to bleached and unbleached human enamel. Journal of Endodontics, $1993 ; 19(3): 112-115$.

22. VIANA PC, et al. Soft tissue waxup and mock-up as key factors in a treatment plan: case presentation. Eur $\mathrm{J}$ Esthet Dent. 2012; 7(3): 310-23.

23. WANG C, et al. Analysis of pulp prognosis in 603 permanent teeth with uncomplicated crown fracture with or without luxation. Dent Traumatol. 2014.

24. WITTMANN K, et al. Restauração estética do sorriso por meio da integração de técnicas de clareamento e restauração direta de resina composta. Clín Int J Bra Dent. 2013; 9:456-467. 